\title{
Dynamic bowel obstruction: aetiology, clinical presentation, management and outcome at Bugando Medical Centre, Mwanza, Tanzania
}

PHILLIPO L. CHALYA*, JOSEPH B. MABULA, ALPHONCE B. CHANDIKA and GEOFREY GIITI

Department of Surgery, Catholic University of Health and Allied Sciences-Bugando, Mwanza, Tanzania

\begin{abstract}
Dynamic bowel obstruction is a common and potentially dangerous surgical emergency with high morbidity and mortality worldwide. No prospective study has been done on this subject in our setting. This study was conducted to describe in our region, the aetiology, clinical presentation, management and outcome of dynamic bowel obstruction. Data were analyzed using SPSS software system. A total of 342 patients were studied. Males outnumbered females by a ratio of 2.1: 1. The median age of patients at presentation was 34 years (range 11 to 78 years). Obstructed hernias (32.7\%) were the commonest cause of dynamic bowel obstruction. Abdominal pain (100\%) and vomiting (86.5\%) were the most frequent presenting symptoms. Thirty-one (9.1\%) patients were HIV positive. Small bowel was the commonest site of obstruction accounting for $89.2 \%$ of cases. Herniorrhaphy was the most frequent surgical procedure performed in 112 (32.7\%) patients. Surgical site infection (38.8\%) was the most common post-operative complication and it was significantly associated with HIV positivity and low CD 4+ count $(p<0.001)$. The overall median of length of hospital stay was 26 days (range 1 to 72 days). Patients who had postoperative complications stayed longer in the hospital and this was statistically significant $(p=0.022$ ). Mortality rate was $14.3 \%$. Delayed presentation, HIV positivity, low CD 4 count ( $<200$ cells $/ \mu \mathrm{l})$, high ASA class and presence of complications were the main predictors of mortality $(p<0.001)$. Obstructed hernias remain the commonest cause of dynamic bowel obstruction in our setting and contribute significantly to high morbidity and mortality. The majority of patients present late when the disease becomes complicated. Early diagnosis and timely definitive treatment are essential in order to decrease the morbidity and mortality associated with this disease.
\end{abstract}

Keywords: bowel obstruction, aetiology, clinical presentation, management, outcome, Tanzania

\section{Introduction}

Dynamic bowel obstruction, a type of bowel obstruction in which the peristalsis is working against an obstructing agent, is s a common surgical emergency globally with high morbidity and mortality (Zahra \& Sultan, 2004; Baloch et al, 2002; Ismail et al. 2005). An estimated $20 \%$ of hospital general surgical emergency admissions are for the management of intestinal obstruction (Baloch et al, 2002). Patient with intestinal obstruction are often seriously ill and require frequent assessment, monitoring of vital signs and clinical progress to determine the need for surgical intervention (Chouhery \& Azam, 2004). Surgical management requires early diagnosis and treatment with meticulous balance of fluids and electrolytes and if appropriate; timely surgical intervention (Macutkiewicz \& Carlson, 2005).

The aetiological pattern of dynamic bowel obstruction has been reported to vary from one geographical area to another and different parts of the same country (Baloch et al, 2002). The most common underlying cause of dynamic bowel obstruction in the West has always been postoperative adhesions as suggested by many reports (Chouhery \& Azam, 2004; Ihedioha et al., 2005; Qureshi et al., 2005; Malik et al., 2010). A number of studies conducted in developing countries had found obstructed/ strangulated hernias to be the most common underlying cause of dynamic intestinal obstruction (Tamijmarane et al., 2000; Wysocki \& Krzywon, 2001; Shittu et al., 2001). With increased awareness among the patients and improved health facilities, the pattern of dynamic (mechanical) bowel obstruction is changing in most developing countries (Ismail et al., 2002). A number of recent studies in developing

* Correspondence: Dr. Phillipo L. Chalya; E-mail: drphillipoleo@yahoo.com 
countries have found adhesive obstruction to be replacing obstructive hernias as the most common cause (Tamijmarane et al., 2000; Markogiannakis et al., 2007; Oladele et al., 2008).

The clinical presentation of dynamic bowel obstruction in most developing countries is not different from those in other studies done in the West (Ihedioha et al., 2005; Qureshi et al., 2005; Malik et al., 2010). However, the majority of patients in developing world present late when the disease becomes complicated (Shittu et al., 2001; Ismail et al., 2005; Qureshi et al., 2005; Malik et al., 2010). Early diagnosis and timely definitive surgical procedure is of paramount in order to improve the chances of survival of patients with this condition. Moreover, the management of dynamic bowel obstruction poses therapeutic challenges to general surgeons practicing in resource-limited countries (Oladele et al., 2008). Late presentation, inadequate preoperative resuscitation and delayed definitive operation are among the hallmarks of the disease in these counties (Qureshi et al., 2005; Malik et al., 2010). Late presentation, inadequate preoperative resuscitation and delayed operation have been found to have a significant effect on prognosis (Adhikari et al., 2010).

The pattern of dynamic bowel obstruction is changing in most developing countries with increased awareness among the patients and improved health facilities (Adesun \& Agba, 1996; Ismail et al. 2005). This makes it essential that studies are made periodically in every region to define the local causes with the idea to do work on their prevention (Adhikari et al., 2010). This study was conducted to describe in our region, the aetiology, clinical presentation, management and outcome of dynamic bowel obstruction.

\section{Methods and Patients}

\section{Study setting and design}

This was a descriptive prospective study of patients operated for dynamic bowel obstruction at Bugando Medical Centre (BMC) from March 2008 to February 2012. BMC is a referral, consultant and teaching hospital for the Catholic University of Health and Allied Sciences-Bugando (CUHAS-Bugando) and other paramedics and it is located in Mwanza city in the north-western part of the United Republic of Tanzania. It is situated along the shore of Lake Victoria and has 1000 beds. The hospital serves as a referral centre for tertiary specialist care for a catchment population of approximately 13 million people from neighbouring regions in north-western Tanzania.

\section{Study population}

All patients with radiological and clinical evidence of dynamic bowel obstruction admitted and operated at BMC were included in this study regardless of the gender of the patients. However, patients aged 10 years and below are usually admitted in the paediatric surgical wards and therefore were excluded from the study. Patients with non-mechanical obstruction and those with irreducible hernia were excluded from the study. Patients who responded to conservative measures were also excluded. Patients who refused to consent for HIV testing were also excluded from the study.

Preoperatively, all the patients recruited into the study had intravenous fluids to correct fluid and electrolyte deficits; nasogastric suction; urethral catheterization and broad-spectrum antibiotic coverage. Relevant preoperative investigations included serum electrolytes, urea and creatinine, blood grouping and cross-matching and ESR. Patients were also screened for HIV testing using Tanzania HIV Rapid Test Algorithm (Lyamuya et al, 2009) and CD 4+ count using FACS or FACSCALIBUR from BD Biosciences USA. A determination of CD 4 count was only performed in HIV positive patients. Radiological investigations including X-ray abdomen erect and supine, X-ray chest PA-view were done in all patients. Abdominal ultrasound was also performed in some patients suspected to have associated abdominal collections. Intraoperative tissue biopsy was taken for histopathological studies. 
After resuscitation all patients under general anaesthesia were subjected to exploratory laparotomy through midline incision. They had pre-operative anaesthetic assessment using the American Society of Anaesthetists (ASA) classification (Wolters et al, 1996) as shown in Table 3. To minimize variability in our study, the assignation of ASA class was performed by a consultant anaesthetist adhering strictly to criteria above. Adequate hydration was indicated by an hourly urine output of $30 \mathrm{ml} /$ hour. The operations were performed either by a consultant surgeon or a senior resident under the direct supervision of a consultant surgeon.

Post-operatively patients were kept nil orally till return of bowl sounds and at that time nasogastric tubes were removed depending on the volume of the nasogastric tube drainage . Parenteral antibiotics were used for a period of up to one week. The postoperative outcome was monitored; patients in ASA classes IV and V were admitted into the intensive care unit after surgery. Final diagnosis and postoperative treatment was dependent on the operative findings and histopathological confirmation in cases of tuberculous bowel obstruction and bowel obstruction due to malignancies. Those found to be tuberculous were started on anti tuberculosis therapy. Data on each patient were entered into a pro forma prepared for the study. The study variables included socio-demographic (i.e. age and sex, cause of obstruction, duration of symptoms, clinical presentation, HIV status, radiological findings, timing of surgical procedure, ASA classification, operative findings and surgical procedure performed. The variables studied in the postoperative period were postoperative complications, hospital stay and mortality. Patients were followed up till discharge or death and thereafter for a period of six-twelve months.

\section{Data analysis}

Statistical data analysis was done using SPSS software version 17.0 (SPSS, Inc, Chicago, IL). Data was summarized in form of proportions and frequent tables for categorical variables and mode and median for continuous variables. P-values were computed for categorical variables using Chi-square $\left(\chi^{2}\right)$ test and Fisher's exact test depending on the size of the data set. Independent student t-test was used for continuous variables. Multivariate logistic regression analysis was used to determine predictor variables that are associated with outcome. A p-value of less than 0.05 was considered to constitute a statistically significant difference.

\section{Ethical consideration}

Ethical approval to conduct the study was obtained from the CUHAS/BMC joint institutional ethic review committee before the commencement of the study. Patients who met the inclusion criteria were requested to sign a written informed consent before being enrolled into the study.

\section{Results}

\section{Demographic data}

During this study period, 342 patients were admitted in the general surgical wards with the diagnosis of dynamic (mechanical) bowel obstruction and underwent surgical intervention. These made the study population. There were $233(68.1 \%)$ males and 109 (31.9\%) females with a male to female ratio of $2.1: 1$. The age of patients at presentation ranged from 11 to 78 years with a median age of 34.0 years. The modal age group was $31-40$ years accounting for $32.2 \%$ of cases (Table 1$)$. Two hundred and six (60.2\%) patients were aged 40 years and below. 
Table 1: Distribution of patients according to age group versus sex

\begin{tabular}{llll}
\hline $\begin{array}{l}\text { Age } \\
\text { years })\end{array}$ & group & (in & \multicolumn{2}{c}{ Sex } & Total (N/\%) \\
\cline { 2 - 3 } & Male $(\mathbf{N} / \%)$ & Female $(\mathbf{N} / \%)$ & $45(13.2)$ \\
$11-20$ & $31(9.1)$ & $14(4.1)$ & $74(21.6)$ \\
$21-30$ & $50(14.6)$ & $24(7.0)$ & $111(32.5)$ \\
$31-40$ & $73(21.4)$ & $38(11.1)$ & $73(21.3)$ \\
$41-50$ & $50(14.6)$ & $23(6.7)$ & $27(7.9)$ \\
$51-60$ & $23(6.7)$ & $4(1.2)$ & $9(2.6)$ \\
$61-70$ & $5(1.4)$ & $4(1.2)$ & $3(0.9)$ \\
$>70$ & $1(0.3)$ & $2(0.6)$ & $342(100)$ \\
Total & $233(68.1)$ & $109(31.9)$ & \\
\hline
\end{tabular}

\section{Aetiology and clinical presentation}

Obstructed hernias were the commonest cause of dynamic bowel obstruction accounting for $32.7 \%$ of cases (Table 2). Majority of patients, 322 (94.2\%) presented with acute bowel obstruction and the remaining 20 (5.8\%) patients presented with chronic bowel obstruction. The duration of symptoms ranged from 1 to 18 days with a median duration of 6.0 days. Twenty $(5.8 \%)$ patients presented within twenty-four hours of onset of symptoms, 42 (12.3\%) between 24 and 48 hours, 58 (17.0\% between 48 and 72 hours and $212(62.0 \%)$ over 72 hours afterwards. The commonest presenting symptom was sudden onset of severe colicky abdominal pain in $342(100 \%)$ patients, followed by vomiting in 296 (86.5\%), constipation in 288 (84.2\%), dehydration, abdominal distension and shock in 202 (59.1\%), 124 (36.3\%) and $42(13.3 \%)$ patients respectively. Previous history of abdominal surgery was reported in $23(6.7 \%)$ patients.

Table 2: Distribution of patients according to the cause of dynamic bowel obstruction

\begin{tabular}{lll}
\hline Cause of obstruction & Number of patients & Percentage \\
\hline Obstructed hernias (external \& internal) & 112 & 32.7 \\
Obstructive bands/adhesions & 64 & 18.7 \\
Volvulus & 58 & 17.0 \\
Malignancies & 42 & 12.3 \\
Intestinal tuberculosis & 32 & $9 \cdot 3$ \\
Intussusceptions & 22 & 6.4 \\
Others & 12 & 3.5 \\
Total & 342 & 100 \\
\hline
\end{tabular}

\section{Investigations}

All patients in this study had plain abdominal $x$-ray films available for review and demonstrated multiple airfluid levels in 328 (95.9\%) patients. Other imaging investigations included abdominal ultrasound, barium enema and abdominal CT scan performed in 26 (7.6\%), 18(5.3\%) and 2(0.5\%) patients respectively. Serum electrolytes revealed hypocalcaemia and hyponatraemia in $96(28.1 \%)$ and 84(24.6\%) patients respectively. Serology test for HIV infection revealed positive results in 31 (9.1\%) patients. Of the HIV positive patients, 10 (32.3\%) patients were known cases on ant-retroviral therapy (ARV) and the remaining 21(67.7\%) patients were newly diagnosed patients. CD 4+ count among HIV positive patients was available in only 26 patients and ranged from 67 cells $/ \mu$ lo 722 cells $/ \mu$ l with the median of 238 cells $/ \mu$ l. A total of nine (34.6\%) HIV positive patients had CD4+ count below 200 cells/ $\mu$ land the remaining 17 (65.4\%) patients had CD4+ count of $\geq 200$ cells $/ \mu$ l. Histopathological examinations revealed malignancies and intestinal tuberculosis in $42(12.3 \%)$ and 32 (9.3\%) patients respectively. 


\section{Admission patterns and pre-operative anaesthetic assessment}

The majority of patients, 302 (88.3\%) were admitted in general surgical wards and the remaining 40 (11.7\%) patients were admitted in the intensive care unit (ICU) where 28 (70.0\%) of them were subjected to ventilator support for a median duration of 8 days (range 2-16 days). All patients were assessed preoperatively using the American Society of Anaesthetists (ASA) pre-operative grading (Table 3) as follows; $125(36.5 \%)$ patients had Grade I, 120 (35.1\%) patients had Grade II, 72(21.1\%) patients had Grade III, 20 (5.8\%) patients hade Grade IV and 5 (1.5\%) patients had Grade V.

Table 3: American Society of Anaesthetists (ASA) classification

\begin{tabular}{ll}
\hline ASA class & Description \\
\hline I & Healthy individual with no systemic disease \\
II & Mild systemic disease not limiting activity \\
III & Severe systemic disease that limits activity but is not incapacitating \\
IV & Incapacitating systemic disease which is constantly life threatening \\
V & Moribund, not expected to survive 24 hours with or without operation \\
\hline
\end{tabular}

\section{Operative findings}

All patients in this study underwent laparotomy. The time interval between the onset of symptoms and definitive surgery ranged from 1 day -20 days with a median of 4 days. Three hundred and thirty-two (97.1\%) patients were operated on emergency bases while 10 (2.9\%) patients had an elective surgery. Three hundred and five (89.2\%) patients had small bowel obstruction while 37 (10.8\%) had large bowel obstruction. Dilated loops of bowel were the most common operative findings in $62.6 \%$ of cases (Table 4 ).

Table 4: Distribution of patients according to operative findings

\begin{tabular}{lll}
\hline Operative findings & Frequency & Percentages \\
\hline Dilated loops of bowel & 214 & 62.6 \\
Obstructed hernias (internal \& external) & 112 & 32.7 \\
Obstructive bands and adhesions & 64 & 18.7 \\
Volvulus \pm ileo-sigmoid knotting & 58 & 17.0 \\
Gangrenous bowel loops & 54 & 15.8 \\
Malignancies & 42 & 12.3 \\
Bowel strictures & 30 & 8.8 \\
Intussusceptions & 22 & 6.4 \\
lleo-caecal mass & 12 & 3.5 \\
Bowel perforations \pm peritonitis & 10 & 2.9 \\
\hline
\end{tabular}

Table 5: Distribution of patients according to surgical procedure performed $(\mathrm{N}=342)$

\begin{tabular}{lll}
\hline Surgical procedure performed & Frequency & Percentage \\
\hline Herniorrhaphy & 112 & 32.7 \\
Release of bands and adhesions & 64 & 18.7 \\
Bowel resection and anastomosis & 62 & 18.1 \\
Right hemicolectomy + ilio-transverse anastomosis & 56 & 16.4 \\
Tumour resection & 42 & 12.3 \\
Colostomy & 26 & 7.6 \\
Pull-through operation & 24 & 7.0 \\
Reduction of intussusceptions & 22 & 6.4 \\
Other surgical procedures & 12 & 3.5 \\
\hline
\end{tabular}

\section{Treatment modalities and outcome}


Herniorrhaphy and release of bands and adhesions were the most common operative procedure in $32.7 \%$ and $18.7 \%$ of cases (Table 5). In this study, a total of 98 post-operative complications were recorded in $88(25.7 \%)$ patients. Of these, surgical site infection was the most common post-operative complication accounting for $38.8 \%$ of cases (Table 6 ). In the present study, the rate of surgical site infection was found to be significantly higher in HIV positive patients than in non HIV patients $(p=0.021)$. Also higher rate of surgical site infection was observed among HIV patients with CD 4+ count below 200 cells $/ \mu \mathrm{l}(p=0.001)$.

Table 6: Distribution of patients according to post-operative complications $(\mathrm{N}=98)$

\begin{tabular}{lll}
\hline Post-operative complications & Frequency & Percentage \\
\hline Surgical site infection & 38 & 38.8 \\
Postoperative pyrexia & 18 & 18.4 \\
Enterocutaneous fistula & 8 & 8.2 \\
Wound dehiscence/ burst abdomen & 7 & 7.1 \\
Pneumonia & 6 & 6.1 \\
Intra-abdominal abscess/ peritonitis & 5 & 5.1 \\
Paralytic ileus & 5 & 5.1 \\
Incisional hernia & 5 & 5.1 \\
Keloids & 4 & 4.1 \\
Urinary tract infections & 2 & 2.1 \\
\hline
\end{tabular}

The overall length of hospital stay (LOS) ranged from 1 to 72 days with a median of 26 days. The median LOS for non-survivors was 4 days (range 1-14days). Patients who had post complications stayed longer in the hospital and this was statistically significant $(p=0.022)$. In this study, forty-nine patients died giving a mortality rate of $14.3 \%$. According to multivariate logistic regression analysis, delayed presentation $(O R=1.3$, $95 \% \mathrm{Cl}$ (1.0- 4.4), $p=0.021)$, HIV positivity (OR $=2.9,95 \% \mathrm{Cl}(3.1-6.9), p=0.022)$, low CD 4 count $(<200$ cells $/ \mu \mathrm{l})$ $(\mathrm{OR}=3.0,95 \% \mathrm{Cl}(1.9-8.5), p=0.000)$, high ASA class (OR = 11.1, 95\% Cl (8.6-12.2), $p=0.004)$, presence of complications $(\mathrm{OR}=1.3,95 \% \mathrm{Cl}(1.1-4.9), p=0.033)$ were the main predictors of mortality.

\section{Follow up of patients}

Of the survivors (293), two hundred sixty-eight (91.5\%) patients were discharged well, twenty (6.8\%) were reoperated for postoperative complications such as peritonitis, burst abdomen and enterocutaneous fistula, and the remaining five (1.7\%) patients were discharged against medical advice. No patient among survivors in this study had permanent disabilities. Of the 293 survivors, one hundred thirty-four (45.7\%) patients were available for follow up at six to twelve months after discharge and the remaining 159 (54.3\%) patients were lost to follow up.

\section{Discussion}

Dynamic (mechanical) obstruction is one of the most common causes for surgical admissions worldwide and contributes significantly to high morbidity and mortality (Ismail et al., 2002; Zahra \& Sultan, 2004; Baloch et al., 2002). In this study, the majorities of patients were in the third decade of life and tended to affect more males than females. Similar findings have been reported in other studies (Ahmed et al., 2001; Zubaidi et al., 2006; Khan et al., 2007). Markogiannakis et al. (2007) reported median age of the patients as 63 years while median age of patients was 25 years in a study conducted by Drozdz et al. (2005). These gross discrepancies may be due to different disease patterns in different geographic regions of the world.

The etiological pattern of dynamic bowel obstruction has been reported in literature to vary from one part of the world to another (Baloch et al., 2002). In Western societies, most of dynamic bowel obstruction are due to postoperative adhesions (Chouhery \& Azam, 2004; Ihedioha et al., 2005; Qureshi et 
al., 2005; Malik et al., 2010), whereas in developing countries, obstructed hernias remain the most common causes of bowel obstruction (Tamijmarane et al., 2000; Wysocki \& Krzywon, 2001; Shittu et al., 2001). In the current study, the main cause of dynamic bowel obstruction was an obstructed hernia. Similar findings have been reported in other developing countries (Tamijmarane et al., 2000; Wysocki \& Krzywon, 2001; Shittu et al., 2001). However, this is contrary to other studies which reported postoperative adhesions as the commonest cause of dynamic bowel obstruction (Chouhery \& Azam, 2004; Ihedioha et al., 2005; Qureshi et al., 2005; Malik et al., 2010). In many developing countries, lack of awareness and financial constraints make many patients present very late with obstructed or strangulated hernia which is a serious life-threatening condition.

The clinical presentation of dynamic bowel obstruction in our patients is not different from those in other studies (Ismail et al., 2002; Qureshi et al., 2005; Kuremu \& Jumbi, 2006; Zubaidi et al., 2006; Chalya et al., 2013), with colicky abdominal pain being common to all the patients. As reported by many authors in developing countries (Shittu et al., 2001; Malik et al., 2010; Chalya et al., 2013), majority of patients in the present study presented late in poor general condition. This was found to be the most important factor influencing the outcome of surgical procedure as also emphasized by a number of authors (Qureshi et al., 2005; Malik et al., 2010; Adhikari et al., 2010). This delayed presentation increases morbidity and mortality many-folds, as is evident from our results. We could not establish the reasons for late presentation in this study.

The prevalence of HIV infection in the present study was 9.1\%, a figure that is significantly higher than that in the general population in Tanzania (6.5\%) (THMIS, 2012). However, failure to detect HIV infection during window period and exclusion of some patients from the study may have underestimated the prevalence of HIV infection among these patients. In a study by Chalya et al. (2013) at the same centre the prevalence of HIV infection among patients with tuberculous bowel obstruction was $21.2 \%$. This difference in HIV seroprevalence in these studies reflects differences in the overall prevalence for risk factors for HIV infection in general population. The authors could not establish the reasons for high HIV seroprevalence in this study. The clinical picture of bowel obstruction may be complex when occurs in association with HIV infection (Iliyasu \& Babashani, 2009). HIV infection has been reported to increase the risk of surgical site infection and mortality (Mawala et al., 2011). In the present study, the rate of surgical site infections and mortality was found to be significantly higher in HIV positive patients than in non HIV patients. Also higher rate of SSI was observed among HIV patients with low CD 4 count $(<200 \mathrm{cells} / \mu \mathrm{l})$.

Emergency surgical intervention is considered to be the standard treatment of choice for patients with dynamic (mechanical) bowel obstruction (Akbar et al., 2010; Chalya et al., 2013). A critical factor in managing these patients is to determine whether patients can be subjected to conservative treatment or to emergency surgery (Chaib et al., 1990). In keeping with other studies (Akbar et al., 2010; Chalya et al., 2013), the majority of patients in this study underwent emergency surgical treatment. One of the many factors affecting the surgical outcome in patients with dynamic bowel obstruction is time interval between duration of onset of bowel obstruction and surgical intervention (Chaib et al., 1990; Adhikari et al., 2010). In the present study, the majority of patients were operated more than 24 hours after the onset of illness. Similar observation was reported by other studies (Shittu et al., 2001; Akbar et al., 2010; Chalya et al., 2013). Delayed definitive surgery in the present study may be attributed to late presentation due to lack of accessibility to health care facilities, lack of awareness of the disease as a result some patients with bowel obstruction may decide to take medications in the pre-hospital period with hope that the symptoms will abate. Late presentation may also result from delayed referral at the peripheral hospitals.

In the present study, herniorrhaphy was the most common surgical procedure performed. Similar results are also described by many authors who have conducted similar study in developing countries (Tamijmarane et al, 2000; Wysocki \& Krzywon, 2001; Shittu et al., 2001). Release of adhesion and bands were the second most common surgical procedure performed. All patients with adhesive obstruction 
were initially given a trial of conservative treatment, and this approach is recommended and adopted by many other authors in their trials (Zahra \& Sultan, 2004; Mehmood et al., 2005; Williams et al., 2005).

The presence of complications has an impact on the final outcome of patients presenting with bowel obstruction. In keeping with other studies (Niaz et al., 2010; Akbar et al., 2010; Chalya et al., 2013), surgical site infection was the most common postoperative complications in the present study. High rate of surgical site infection in the present study is attributed to HIV seropositivity and low CD 4 count.

The overall median duration of hospital stay in the present study was 26 days which is higher than that reported by other authors (Manzoor \& Muhammad, 1999; Niaz et al., 2010; Chalya et al., 2013). This can be explained by the presence of large number of patients with postoperative complications in our study. However, due to the poor socio-economic conditions in Tanzania, the duration of inpatient stay for our patients may be longer than expected. The overall mortality rate in this study was $14.3 \%$ and it was significantly associated with delayed presentation, HIV positivity, low CD 4 count, high ASA class and presence of complications. Addressing these factors responsible for high mortality in our patients is mandatory to be able to reduce mortality associated with this disease.

Self discharge by patient against medical advice is a recognized problem in our setting. Similarly, poor follow up visits after discharge from hospitals remain a cause for concern. These issues are often the results of poverty, long distance from the hospitals and ignorance. Delayed presentation and the large number of loss to follow up were the major limitations in this study. Since our institution is a tertiary referral hospital, we mainly attended to cases which could not be managed under the primary or secondary level of health care; therefore, an accurate etiological assessment of acute intestinal obstruction might not have been reflected in our study. Also, most of our patients were from a poor socioeconomic status with a high prevalence of malnutrition; therefore, the morbidity and mortality are likely to be higher. The higher number of terminally ill patients as well as patients with AIDS also led to a higher mortality rate in our study. Despite these limitations in our study, it reveals that obstructed hernia rather than adhesions is the most common cause for dynamic intestinal obstruction in this part of Tanzania.

In conclusion, this study demonstrates that the pattern of dynamic bowel obstruction differs from the Western world with obstructed hernias being the most important cause in this region and contributes significantly to high morbidity and mortality. It also highlights the necessity of using universal precautions because of the ever increasing number of HIV patients in those with bowel obstruction. The majority of patients in our environment present late when the disease becomes complicated. Early diagnosis, aggressive resuscitation and timely definitive treatment are essential in order to decrease the morbidity and mortality associated with this disease.

\section{Acknowledgements}

The authors would like to thank the members of staff of the Department of Surgery of Bugando Medical Centre for their invaluable contributions in the preparation of this manuscript. Special thanks go to the Senior House Officers of the Department of Surgery for their support in data collection and contribution in the management of our study patients.

\section{References}

Adesun, K.A.R. \& Agba, K.F.A. (1996) The changing pattern of acute intestinal obstruction in tropical African population. East African Medical Journal 73, 727-31

Adhikari, S., Hossein, M.Z., Das, A., Mitra, N. \& Ray, U. (2010) Etiology and outcome of acute intestinal obstruction: A review of 367 patients in Eastern India. Saudi Journal of Gastroenterology 16, 285-7. 
Akbar, M., Islam, F., Haider, I.Z., Naveed, D., Akbar, I., Khattak, I., Akbar, K. \& Zafar A (2010) Surgical management of tuberculous small bowel obstruction. Journal of Ayub Medical College Abbottabad 22, 171-175.

Baloch, N.A., Babar, K.M., Mengal, M.A. \& Babar, S.A.A. (2002) Spectrum of Mechanical Intestinal Obstruction. Journal of Surgery Pakistan 7, 7-9.

Chaib, E., Toniolo, C.H., Figueira, N.C., Santana, L.L., Onofrio, P.L. \& de Mello, J.B. (1990) Surgical treatment of intestinal obstruction. Arg Gastroenterology 27, 182-6.

Chalya, P.L., Mchembe, M.D., Mshana, S.E., Rambau, P., Jaka, H. \& Mabula, J.B. (2013) Tuberculous bowel obstruction at a university teaching hospital in Northwestern Tanzania: a surgical experience with 118 cases. World Journal of Emergency Surgery 8, 12.

Chouhery, A.K. \& Azam, M. (2004) An etiological spectrum of mechanical intestinal obstruction. Pakistan Armed Forces Medical Journal 54, 19-24.

Drozdz, W., Lejman, W. \& Tusiński, M. (2005) Mechanical bowel obstruction. Surgical problem at the turn of the XIX-XX century, and the XX-XXI century. One institutional experience. Przeg Lek 62, 105-110.

Ihedioha, U., Alani, A., Modak, P., Chong, P. \& O'Dwyer, P.J. (2006) Hernias are the most common cause of strangulation in patients presenting with small bowel obstruction. Hernia 10, 338-340.

Iliyasu, Z. \& Babashani, M. (2009) Prevalence and predictors of tuberculous co-infection among HIV seropositive patients attending the Aminu Kano Teaching Hospital, northern Nigeria. Journal of Epidemiology 19, 81-87.

Ismail, Khan, M., Shah, S.A. \& Ali, N. (2005) Pattern of dynamic Intestinal Obstruction in adults. Journal of Postgraduate Medicine 19, 157-61.

Kuremu, R.T. \& Jumbi, G. (2006) Adhesive intestinal obstruction. East African Medical Journal 83, 333-336.

Lyamuya, E.F., Aboud, S., Urassa, W.K., Sufi, J., Mbwana, J., Ndungulile, F. \& Massambu, C. (2009) Evaluation of rapid HIV assays and development of National Rapid HIV test algorithms in Dar es Salaam, Tanzania. BMC infectious Diseases 9, 19.

Macutkiewicz, C. \& Carlson, G.L. (2005) Acute Abdomen: Intestinal obstruction. Surgery International 70, 1014.

Manzoor, A. \& Muhammad, A.M. (1999) Pattern of mechanical intestinal obstruction in adults. Journal of the College of Physicians and Surgeons 9, 441-443.

Markogiannakis, H., Messaris, E., Dardamanis, D., Pararas, N., Tzertzemelis, D. \& Giannopoulos P. (2007) Acute mechanical bowel obstruction: Clinical presentation, etiology, management and outcome. World Journal of Gastroenterology 13, 423-437.

Mawalla, B.M., Mshana, S.E., Chalya, P.L., Imirzalioglu, C. \& Mahalu, W. (2011) Predictors of surgical site infections among patients undergoing major surgery at Bugando Medical Centre in Northwestern Tanzania. BMC Surgery 11, 21.

Mehmood, Z., Aziz, A., Iqbal, M., Sattar, I. \& Khan A. (2005) Causes of intestinal obstruction: A study of 257 patients. Journal of Surgery Pakistan 10, 17-19.

Oladele, A.O., Akinkuolie, A.A. \& Agbakwuru, E.A. (2008) Pattern of intestinal obstruction in a semi urban Nigerian hospital. Nigerian Journal of Clinical Practice 11, 347-350.

Qureshi, M.I., Anwar, I., Dar, H.M., Ahmad, A. \& Durrani, K.M. (2005) Managing small intestinal obstruction: Proceeding Shaikh Zayed Postgraduate Medical Institute 19, 19-23.

Shittu, O.B., Gana, J.Y., Alawale, E.O. \& Ogundiran, T.O. (2001) Pattern of mechanical intestinal obstruction in Ibadan: a 10 year review. African Journal of Medical Science 30, 17-21.

Tamijmarane, A., Chandra, S. \& Smile, S.R. (2000) Clinical aspects of adhesive intestinal obstruction. Tropical Gastroenterology 21, 141-143. 
THMIS (2012) Tanzania HIV/AIDS and Malaria Indicator Survey 2011-12. Tanzania Commission for AIDS (TACAIDS), Zanzibar AIDS Commission (ZAC), National Bureau of Statistics (NBS), Office of the Chief Government Statistician (OCGS), and ICF International (2013), Dar es Salaam, Tanzania

Williams, S.B., Greenspon, J., Young, H.A. \& Orkin, B.A. (2005) Small bowel Obstruction: Conservative versus surgical management. Diseases of Colon and Rectum 48, 1140-1146.

Wolters, U., Wolf, T., Stutzer, H. \& Schroder, T. (1996) ASA classification and perioperative variables as predictors of postoperative outcome. British Journal of Anesthesia 77, 217-222.

Wysocki, A. \& Krzywon, J. (2001) Causes of intestinal obstruction. Przegl Lek. 58, 507-508.

Zahra, T. \& Sultan, N. (2004) Prevalence of Intestinal T.B amongst cases of bowel obstruction. Pakistan Journal of Surgery 20, 82-85.

Zubaidi, A., Al-Saif, F. \& Silverman, R. (2006) Adult intussusceptions: a retrospectives review. Diseases of Colon and Rectum 49, 1546-1551, 